\title{
REFLECTION OF A WEAK DISCONTINUITY OF THE AXIS AND THE PLANE OF SYMMETRY
}

\author{
Bulat Pavel Viktorovich \\ Saint-Petersburg National Research University of Information Technologies, \\ Mechanics and Optics, Saint-Petersburg, Russia
}

Received 2014-03-31; Revised 2014-04-10; Accepted 2014-04-17

\begin{abstract}
In this article we consider the problem of discontinuous characteristic (weak discontinuity) reflection from the axis and the plane of symmetry of the supersonic flow. There is a known problem of perturbations radial focusing when during numerical calculations reflection of a weak perturbation wave from the axis of symmetry leads to an abrupt change in the distribution of dynamics variables along the axis, i.e., to a strong discontinuity, which seems unphysical. By an asymptotic expansion in the vicinity of the axis of symmetry the expression for the intensity of a weak discontinuity was found before the point of intersection with the axis as well as beyond it. It is shown that the appearance of strong discontinuity in the results of numerical calculations is a computing feature, which is a consequence of the difference approximation of the equations. A solution for changes in the intensity of a weak discontinuity as it approaches the axis or plane of symmetry in the plane or axisymmetric supersonic flow was obtained as well.
\end{abstract}

Keywords: Weak Discontinuity, Discontinuous Characteristic, Perturbations Radial Focusing Paradox, Supersonic Flow, Intensity of a Weak Discontinuity, Method of Characteristics

\section{INTRODUCTION}

It is important to determine the intensity of discontinuous characteristics (weak discontinuities) in a number of problems of supersonic and hypersonic aerodynamics, because their amplification may lead to the emergence of shoch waves within the flow. At the symmetry axis curvature of discontinuities and their intensity increases strongly as they approach the axis, so the problem is particularly acute. The problem of studying of the interaction of simple waves, the first and last characteristics of which are discontinuous, between themselves, with weak discontinuities, solid walls and shock waves arises at designing of supersonic and hypersonic air inlets, combustion chambers with detonation combustion and acoustic wave resonators. In recent years close attention was paid to this area. This problem was considered in works under the direction of Uskov and Chernyshev (2014a). So the solution for supersonic flow in the vicinity of the nozzle lip described in his "Twodimensional over-expanded jet flow parameters in supersonic nozzle lip vicinity" Silnikov et al. (2014). This study considers conditions leading to the emergence of a hanging shock wave. The solutions for a Prandtl-Meyer plane wave interaction with two-dimensional shock wave were given in works of Uskov and Chernyshev (2014b). During the research work of past 15 years the problem of interaction of simple one-dimensional waves and their reflection from a solid wall was considered by Arkhipova and Uskov (2012). Arkhipova and Uskov (2013) found as well a solution for a Prandtl-Mayer plane wave reflection from a solid wall.

Axisymmetric flows are more difficult in terms of analytical solutions obtaining, as presence of axial symmetry leads to curvature of characteristics. Dynamic compatibility conditions on low break and the general problem of characteristics interaction calculation with considering of the non-uniformity of flow were formulated by Uskov and Mostovykh (2011).

\section{THE INITIAL SYSTEM OF EQUATIONS}

Flows of ideal gas are described by the system of Euler equations. It is convenient for later use to write this system in the coordinates $n$ (the length of the 
normal to the streamlines) -s (arc length along the current line) using the basic gasdynamic irregularities $\mathrm{N}_{\mathrm{i}}$ (Bulat and Bulat, 2013) Equation 1:

$N_{1}=\frac{\partial \ln P}{\partial s}, N_{2}=\frac{\partial \vartheta}{\partial s}, N_{3}=\varsigma=\frac{\partial \ln P_{0}}{\partial n}$

Where:

$P=$ Pressure

$\vartheta=$ Angle of the velocity vector

$P_{0}=$ Stagnation pressure

$\zeta=$ Vorticity

After simple transformations, we obtain a system of Equation 2:

$$
\begin{aligned}
& \frac{M^{2}-1}{\gamma M^{2}} N_{1}+\frac{\partial \vartheta}{\partial n}+\frac{\sin \vartheta}{y}=0, \\
& \gamma M^{2} \frac{\partial \ln V}{\partial s}=-N_{1}, \\
& M^{2} N_{2}=-\frac{\partial \ln P}{\partial n} .
\end{aligned}
$$

Where:

$M=$ Mach number

$V=$ Velocity

$\gamma=$ Adiabatic index

Sometimes the system of equations in the variables n$\mathrm{s}$ is called Euler equations in natural coordinates. However, this is not true, because unit vectors $s$ and $n$ form orthogonal geodesic lines of the phase space, but do not form the coordinate system in the usual sense of the length along the lines of $\mathrm{s}$ and $\mathrm{n}$. Indeed, as we move along the coordinate $x_{i}, x_{j}$ coordinate must not be changed. For natural coordinate system it is valid only in a particular case, such as flow from source.

In the study of isentropic compression and expansion waves as well as geometry characteristics (the concept of characteristics will be introduced below) it is convenient to rewrite the system (2) using a function of the Prandtl-Mayer:

$$
\begin{aligned}
& \operatorname{ctg} \alpha \frac{\partial \omega}{\partial s}-\frac{\partial \vartheta}{\partial n}=\frac{\delta}{y} \sin \alpha, \\
& \operatorname{ctg} \alpha \frac{\partial \vartheta}{\partial s}-\frac{\partial \omega}{\partial n}=\frac{\zeta}{\gamma} \operatorname{ctg} \alpha \sin ^{2} \alpha, \\
& \operatorname{ctg} \alpha=\sqrt{M^{2}-1}, \\
& \omega=\frac{1}{\sqrt{\varepsilon}} \operatorname{arctg}\left(\sqrt{\varepsilon\left(M^{2}-1\right)}\right)-\operatorname{arctg} \sqrt{M^{2}-1}
\end{aligned}
$$

\section{THE FIRST DISCONTINUITY CHARACTERISTIC OF THE RAREFACTION WAVE}

As you know, gas dynamics equations do not impose conditions on changing of the parameters normal to the gas-dynamic characteristics, so unevenness $\mathrm{N}_{\mathrm{i}}$ before characteristic and after it may not coincide. Such characteristics are called discontinuous or weak discontinuities. An important parameter characterizing a weak discontinuity is its intensity. An intensity is taken to mean the difference $\left[\mathrm{N}_{\mathrm{i}}\right]=\hat{\mathrm{N}}_{\mathrm{i}}-\mathrm{N}_{\mathrm{i}}$, for example, the difference between the curvature of the streamlines. Crossing the shock wave discontinuous characteristic change its curvature. Reflecting from axis a weak discontinuity changes the distribution of the parameters along the axis. The intensity depends on the discontinuous characteristics geometry and conditions at its inception. Let us consider intensity of the first characteristics of a centered rarefaction wave in a uniform stream.

\subsection{Prandtl-Meyer Plane Wave}

In a plane rarefaction wave discontinuous are the first and last characteristics. Let the nozzle expires uniform flow $\left(\mathrm{N}_{1}=\mathrm{N}_{2}=0\right)$. Let us introduce a polar coordinate system associated with the edge of the nozzle. For an arbitrary curve in polar coordinates following conditions are true (Fig. 1a):

$$
\frac{r^{\prime}}{r}=\frac{1}{r} \frac{d r}{d \varphi}=\operatorname{tg}(\varphi+\psi) ; \operatorname{tg} \varphi=\frac{d r}{d \varphi}
$$

Where:

$\mathrm{r}=$ Radius vector

$\varphi=$ Polar angle measured from the vertical axis

$\psi=$ Angle curve slope to the axis $\mathrm{x}$

\subsection{For Streamline $\psi=0$}

For the discontinuous characteristics of the flock $(\chi= \pm 1)$ following conditions occur:

$$
\psi_{+}=\pi / 2-\varphi_{1}, \psi_{-}=\pi / 2-(\varphi+2 \alpha)
$$

From these relations we obtain the differential equations of the streamlines and characteristics in Prandtl-Meyer wave: 


$$
\begin{aligned}
& r^{\prime}=r c t g \alpha,- \text { streamline } \\
& \varphi=\text { const },-v_{+} \\
& r^{\prime}=r c t g 2 \alpha,-v_{-}
\end{aligned}
$$

Integrating this system, we obtain the well-known equation for the streamline in a plane rarefaction wave:

$$
\begin{aligned}
& \frac{r_{s}}{r_{0}}=\left|\frac{\cos Z_{0}}{\cos Z}\right|^{\frac{1}{\varepsilon}}, Z=\sqrt{\varepsilon}(\varphi+C), C \\
& =\frac{1}{\sqrt{\varepsilon}} \operatorname{arctg}\left(\sqrt{\varepsilon} \operatorname{ctg} \alpha_{0}\right)+\alpha_{0}-\frac{\pi}{2}
\end{aligned}
$$

Curvature of an arbitrary curve in polar coordinates described by the equation:

$$
K_{s}=\frac{1+2\left(r^{\prime} / r\right)^{2}-r^{2}}{r\left(1+\left(r^{\prime} / r\right)^{2}\right)^{3 / 2}}
$$

Which leads to the following expression for the curvature of the streamlines in the Prandtl-Meyer wave:

$$
\frac{\partial \vartheta}{\partial S}=\frac{1-\varepsilon}{r} \cos ^{2} \alpha_{0} \sin \alpha_{0}=-\frac{(1-\varepsilon)\left(M_{0}^{2}-1\right)}{r M_{0}^{3}}
$$

where, parameters in the undisturbed flow marked by the index " 0 ". From the Equation 3 it follows that the intensity of discontinuous characteristics varies inversely with $r$, becomes infinite at the center of the wave and on the plane of symmetry equals following:

$$
\frac{\partial \vartheta}{\partial S}=\frac{1-\varepsilon}{1-y} \cos ^{2} \alpha_{0} \sin ^{2} \alpha_{0}=-\frac{(1-\varepsilon) \sin ^{2} 2 \alpha_{0}}{2(1-y)}
$$

\subsection{Axisymmetric Rarefaction Wave in a Uniform Stream}

In a uniform flow first discontinuous characteristic of centered rarefaction wave is rectilinear. Let us consider nearest characteristic $\mathrm{v}_{-2}$ of the same direction, which lies inside the wave. At the center of wave (at the edge of the nozzle), these characteristics differ by a small angle $\Delta \varphi$ (Fig. 1b). From the condition on the characteristic $\mathrm{v}_{+}$:

$$
\frac{d(\omega-\vartheta)}{d v_{+}}=\frac{\sin \vartheta}{y} \sin \alpha
$$

It follows that in the vicinity of discontinuous characteristic $\mathrm{d} \omega=\mathrm{d} \vartheta$, i.e., following expression is valid:

$$
\omega_{2}-\omega_{1}=\vartheta_{2}
$$

It can be shown that $\omega$ and $\vartheta$ on characteristic $v_{+}$ associated by the same way as in the Prandtl-Meyer flow:

$$
\begin{aligned}
& \omega_{2}^{a}-\omega_{2}=\omega_{2}^{a}-\omega_{1}-\left(\omega_{2}-\omega_{1}\right) \\
& =\vartheta_{2}^{a}-\vartheta_{1}-\left(\vartheta_{2}-\vartheta_{1}\right)=\vartheta_{2}^{a}-\vartheta_{2}
\end{aligned}
$$

where, parameters in the corner point A marked by the index "a".

Conditions on $\mathrm{v}_{+}$can be written as;

$$
2 \frac{d \vartheta}{d v_{-}}=2 \frac{d \omega}{d v_{-}}=\frac{\sin \vartheta}{y} \sin \alpha
$$

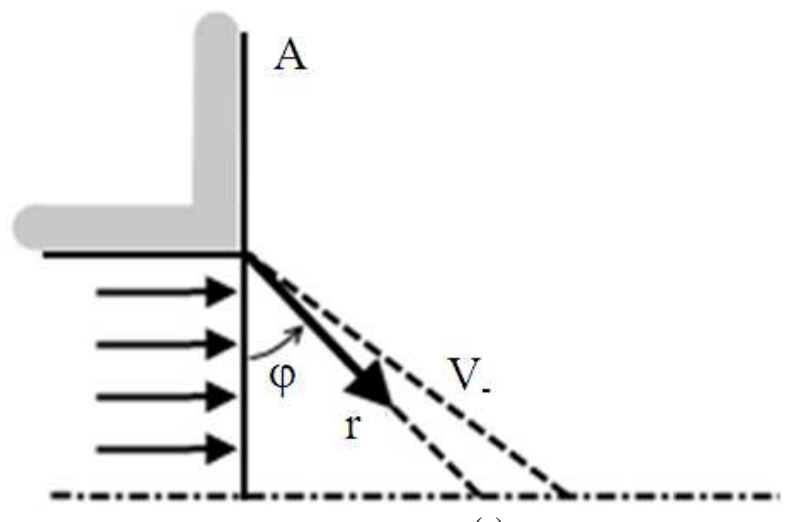

(a)

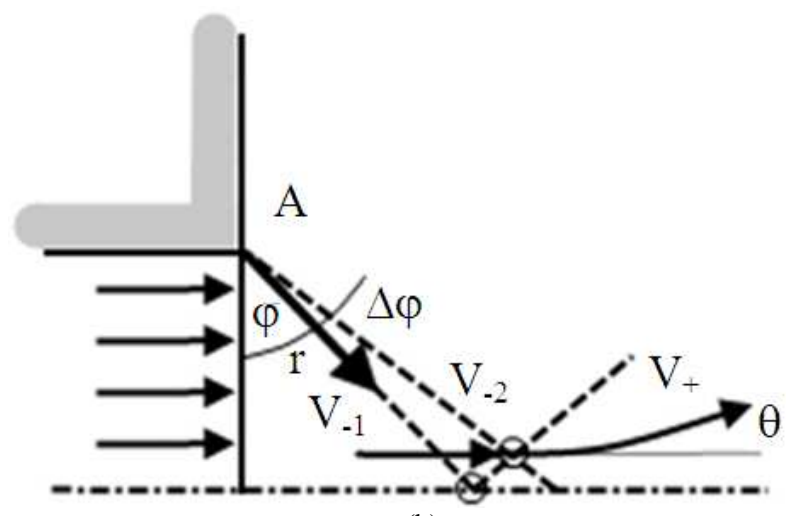

(b)

Fig. 1. On the calculation of the intensity of discontinuous characteristic in centered rarefaction wave (a) plane wave (b) axisymmetric 
Separation of variables leads to the differential equation:

$$
\begin{aligned}
& \frac{\sin \vartheta \cos \alpha d \vartheta}{\sin \vartheta \sin \alpha}=\frac{\sin \alpha \cos \vartheta d \vartheta}{\sin \vartheta \sin \alpha} \\
& =\frac{d y}{2 y} \Leftrightarrow \operatorname{ctg} \alpha d \omega-\operatorname{ctg} \vartheta d \vartheta=\frac{d y}{2 y}
\end{aligned}
$$

Whose integration on the assumption that the Mach angle is changed a little and $\mathrm{d} \varphi$ tends to zero, leads to the following expression Equation 4:

$$
\Delta \vartheta=\frac{(1-\varepsilon) \cos ^{2} \alpha d \varphi}{\sqrt{y}(1-\varepsilon) \cos ^{2} \alpha \operatorname{ctg} \alpha d \varphi}
$$

Let us now consider the increment of the arc along the streamline $\Delta \mathrm{S}$. Due to smallness of $\Delta \varphi$, curvilinear triangle $\mathrm{ABC}$ can be calculated by formulas of a rectilinear triangle, then Equation 5:

$$
\Delta S=\frac{d \varphi(1-y)}{\sin ^{2} \varphi-0.5 d \varphi \sin 2 \varphi}
$$

In order to obtain a discontinuous characteristic intensity, we should divide (4) by (5). Neglecting terms containing $\mathrm{d} \varphi$ we obtain an equation that determines intensity of a weak discontinuity:

$$
\left[\frac{\partial \vartheta}{\partial S}\right]=(1-\varepsilon) \frac{\cos ^{2} \alpha \sin ^{2} \alpha}{\sqrt{y}(1-y)}
$$

Analysis of the extremum of the last equation:

$$
(1-\varepsilon) \frac{\cos ^{2} \alpha \sin ^{2} \alpha}{y(1-y)^{2}}\left(\frac{1-3 y}{2 \sqrt{y}}\right)=0
$$

Shows that the minimum intensity of the discontinuous characteristic lies at a distance of $1 / 3$ from the axis of symmetry.

\section{PERTURBATIONS RADIAL FOCUSING PARADOX}

During the construction of numerical methods often arises the problem of computing features in the area of weak discontituity reflection from the symmetry axis. This phenomenon is known as
Perturbations radial focusing paradox. Often as a result of the difference simulation of supersonic flow of an ideal gas it turns out that at the point of reflection of the rarefaction wave first characteristic from the axis derivative $\partial \omega / \omega S$ tends to infinity (Fig. 2). As a result, the solution becomes unstable, oscillations of parameters and other unwanted effects appear.

At first glance the possible existance of this paradox is indicated by the theorem on the continuity of U-function (Adrianov et al., 1995). Indeed, before the first characteristic flow is uniform, pressure gradient and curvature of the streamlines on the axis are equal to zero. Before the first discontinuous characteristic $\partial \omega / \omega S>0$, but the curvature of the streamline remains zero. It seems there is a violation of the theorem on the U-function, which can only happen when a weak discontinuity in the longitudinal pressure gradient tends to infinity (Fig. 2, the graph below).

In fact, there is no paradox. Let us turn to Fig. 3. It can be seen that the flow in the rarefaction wave on the axis of symmetry begins to accelerate, breaking two weak discontinuity in different directions: The incident and reflected characteristics. Gradient on the axis defined by the relation:

$\frac{\partial \hat{\omega}}{\partial S}=-\frac{3}{2} \frac{1-\varepsilon}{y_{a}} \sin ^{2} \alpha_{0} \cos ^{2} \alpha_{0}$

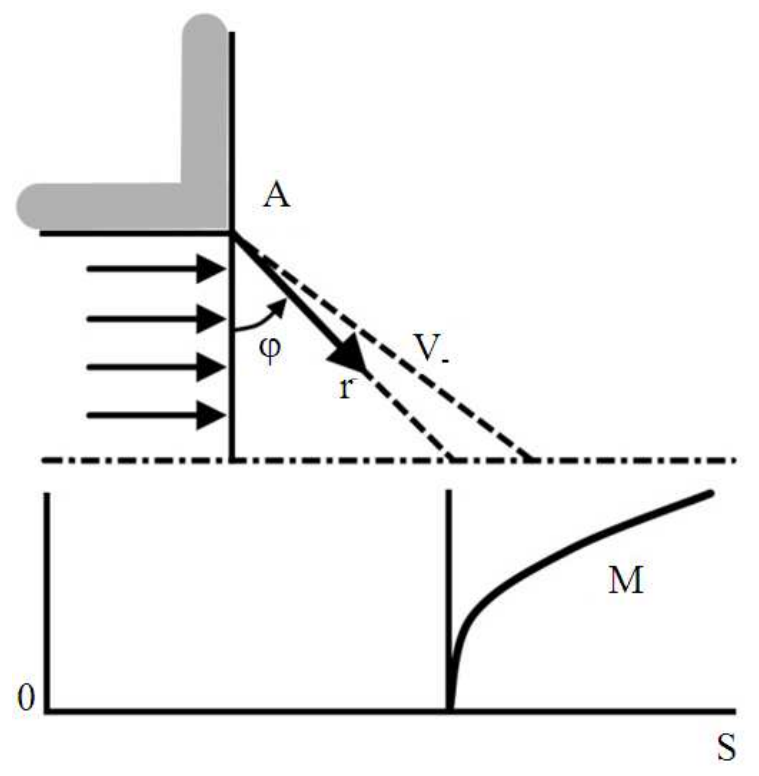

Fig. 2. Perturbations radial focusing paradox 


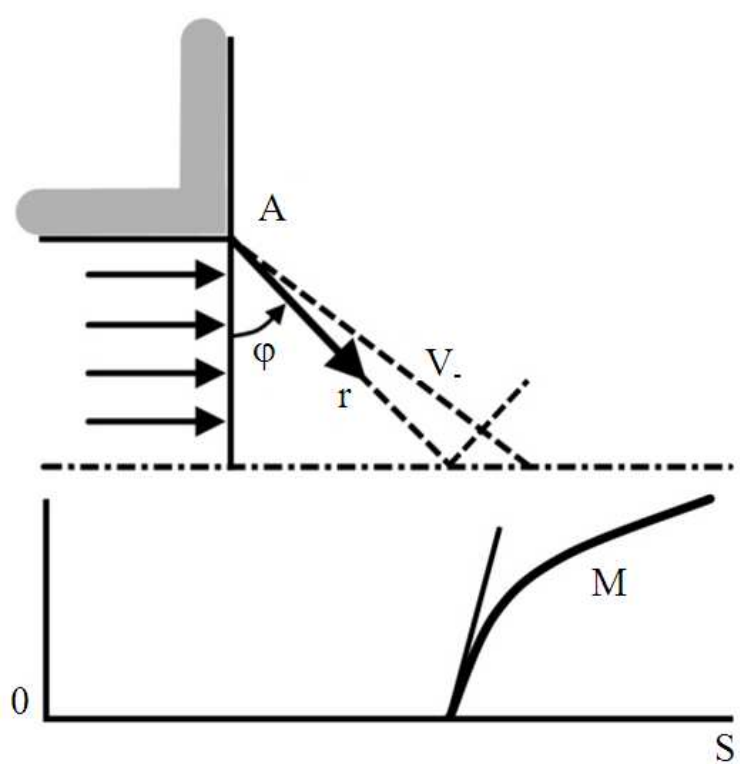

Fig. 3. Reflection of a weak discontinuity from the axis of symmetry

Discontinuity of the streamline curvature value is also obtained from Equation 6, but first with the sign "+" when passing through characteristic $\mathrm{v}_{-}$, then with the sign "-" when passing through the characteristic of the second family $v_{+}$. As a result, the curvature of streamline on the axis remains zero. Thus, perturbations radial focusing paradox does not exist, the gradient of the Mach number on the axis remains finite (Fig. 3), but the second derivative really becomes infinite. At the intersection point of characteristics $\mathrm{v}_{\text {- }}$, which is close to the first discontinuous characteristic of the rarefaction wave, with the reflected characteristic $\mathrm{v}_{+}$singularities does not occur. Curvature of the streamline characteristics after the reflection point of the characteristic from the axis remains zero, but its derivative takes a positive value, which causes a streamline deviation from the axis (Fig. 3), the pressure drops and the flow accelerates.

\section{CONCLUSION}

The concept of the weak discontinuity was introduced. We examined the basic relations on the first discontinuous characteristic of plane and axisymmetric centered rarefaction wave. During the mathematical transformations it was shown that the intensity of the first discontinuous characteristics of centered rarefaction wave in an axisymmetric uniform flow has an extremum at a distance of $1 / 3$ of the plane of symmetry. We as well considered well-known discontinuity radial focusing paradox. Показано, чтоIt should be classified to the computing features, arising during the difference modelling of an ideal gas flows.

\section{FINDINGS}

The analytical solutions for discontinuous characteristics in axisymmetric supersonic flow of an ideal gas allow explicitly allocate conditions of the hanging jumps occurrence in supersonic flows. It matters not only in the problems of ideal gas calculation, but generally wherever occurrence of strong discontinuities in smooth supersonic flow is important. For example, in the nonstationary case an appearance of a detonation wave during combustion can be attributed to such tasks. Another important fact is proof that the perturbations radial focusing paradox doesnt have a physical meaning. Earlier this paradox was referred to the features of an ideal inviscid gas equations solutions and it was believed that the transition to the Navier-Stokes removes this paradox and features emerging on axis are a manifestation of some physical phenomena or disadvantages of differential turbulence models. However, it turned out that it is not so. The paradox is a consequence of the difference approximation of the initial system of equations, so its exclusion from the calculation results requires careful meshing at the axis of symmetry.

\section{ACKNOWLEDGEMENT}

This article was prepared as part of the "1000 laboratories" program with the support of SaintPetersburg National Research University of Information Technologies, Mechanics and Optics.

\section{REFERENCES}

Adrianov, A.L., A.L. Starykh and V.N. Uskov, 1995. Interference of stationary gas-dynamic discontinuities. Novosibirsk: Nauka. ISBN: 9785020306776.

Arkhipova, L.P. and V.N. Uskov, 2012. Reflection of centered Riemann rarefaction wave with supersonic trailing edge from solid and smooth wall. Vestnik St. Petersburg University. 
Arkhipova, L.P. and V.N. Uskov, 2013. Universal solution to the problem of reflection of onedimensional traveling waves from a solid surface and their analysis for compression waves. Vestnik St. Petersburg University.

Bulat, P.V. and M.P. Bulat, 2013. The analysis centric isentropic compression waves. World Applied Sci. J., 27: 1023-1026. DOI: 10.5829/idosi.wasj.2013.27.08.13719

Silnikov, M.V., M.V. Chernyshov and V.N. Uskov, 2014. Two-dimensional over-expanded jet flow parameters in supersonic nozzle lip vicinity. Acta Astronaut., 97: 38-41. DOI: 10.1016/j.actaastro.2013.12.010
Uskov, V.N. and M.V. Chernyshov, 2014a. Analytical solutions for Prandtl-Meyer wave-oblique shock overtaking interaction. Acta Astronaut., 99: 175183. DOI: $10.1016 /$ j.actaastro.2014.02.025

Uskov, V.N. and M.V. Chernyshov, 2014b. The interaction of Prandtl-Meyer wave with the oblique shock of the same direction. J. Energy Power Eng., 8: 121-136.

Uskov, V.N. and P.S. Mostovykh, 2011. Compatibility conditions on weak discontinuity in the axisym metric flow of inviscid gas. Vestnik St. Petersburg University. 